\title{
Replik auf Ritschard zur Qualität der Mammographieprogramme
}

\author{
Jacques Fracheboud ${ }^{a}$, Jean-Luc Bulliard ${ }^{b}$, Marcel Zwahlenc $^{c}$ \\ a Eidgenössisch diplomierter Arzt, Erasmus University Medical Center, Rotterdam; ' PD Dr phil., Institut universitaire de médecine sociale et préventive, \\ Lausanne; ' Prof. Dr. phil., Institut für Sozial- und Präventivmedizin, Bern
}

Die Autoren des Monitoringberichts 2011 der Mammographieprogramme in der Schweiz gehen auf die kürzlich geäusserten Zweifel an deren Qualität ein. Für sie sind diese Zweifel nicht nachvollziehbar, wenn man die Kennzahlen des Monitoringberichts 2011 detaillierter mit den empfohlenen Werten in den Europäischen Richtlinien vergleicht. Es ist eine Stärke der Programme, dass sie regelmässig über die erreichten Resultate Rechenschaft ablegen.

Mit dem Artikel «Mangelnde Qualität der Mammographiescreening-Programme?» in Ausgabe 49/2015 [1] bezweifelt der Autor die Qualität der Brustkrebsfrüherkennungsprogramme in der Schweiz. Er tut dies aufgrund der Ergebnisse des zweiten gesamtschweizerischen Monitoringberichts der Mammographieprogramme 2011 [2], die er mit der «internationalen Literatur» verglich.

Bei genauerem Hinsehen zeigt sich aber, dass die im Monitoringbericht enthaltenen Ergebnisse nicht mit den faktischen Resultaten anderer Mammographie-

Ritschard unterscheidet nicht präzise zwischen Prävalenzscreenings und Folgescreenings.

programme oder Evaluationsstudien verglichen wurden, sondern mit zum besseren Verständnis vereinfachten und gerundeten «natürlichen Häufigkeiten». Diese Häufigkeiten und Annahmen über Sensitivität und Spezifität der Mammographie wurden so bereits in den Arbeiten von Gerd Gigerenzer über Risiko-Verständnis und Risiko-Kommunikation publiziert; Herr Ritschard war bei einer Arbeit Mitautor [3]. Der Verfasser vergleicht in der Folge eigene Berechnungen auf der Grundlage dieser früheren Arbeiten mit den im Schweizer Monitoringbericht 2011 veröffentlichten Resultaten und vermutet, dass die Qualität des Mammographiescreenings «in der Schweiz möglicherweise ungenügend sein könnte».

Bei den real stattfindenden MammographiescreeningProgrammen ist es wichtig, erstmalige und nachfolgende Screenings zu unterscheiden. Die Brustkrebsentdeckungsraten 2011 waren mit 5,8 per 1000 erstuntersuchten Frauen (Prävalenzscreening) und 5,1 per 1000 Folgeuntersuchungen etwas niedriger als im Jahr 2010. Diese Raten beruhen allerdings auf relativ kleinen Zahlen, nämlich 106 (bzw. 238) Brustkrebsdiagnosen bei 18148 Prävalenzscreenings (bzw. 46312 Folgescreenings), was zu Resultaten mit einer deutlichen statistischen Unsicherheit führt (95\% Konfidenzintervall von 4,8-7,1 pro 1000 für das Prävalenzscreening und 4,5-5,8 pro 1000 für Folgescreenings). Die Brustkrebsentdeckungsraten mit ihrer statistischen Unsicherheit liegen aber durchaus im Rahmen derjenigen von anderen nationalen Mammographieprogrammen wie in Deutschland, Italien, Frankreich oder den Niederlanden [4-7]. Keines dieser Programme weist übrigens eine Brustkrebsentdeckungsrate von 9 pro 1000 erstuntersuchten Frauen aus, wie von Ritschard erwartet.

Um die Falsch-Negativrate (Intervallkarzinomrate) zu bestimmen, muss die wirkliche Prävalenz des vor dem Screening bisher unerkannten Brustkrebses und die wirkliche Sensitivität der Screening-Mammographie bekannt sein. Beide allerdings sind deutlich altersabhängig. Addiert man die Brustkrebsentdeckungsraten und die Häufigkeit von Intervallkarzinomen, so erhält man eine bessere Annäherung an die Prävalenz, wobei nicht garantiert ist, dass sich falsch-negative Mammographien zwischen zwei Screenings als Intervallkarzinom manifestieren. Die Mammographieprogramme der Kantone Genf, Waadt und Wallis der Jahre 19992006 zeigten eine Rate von Intervallkarzinomen zwischen 1,9 und 2,5 pro 1000 [8] nach Prävalenzscreenings. Somit wäre mit einer Brustkrebsprävalenz von 0,8\% bis 0,9\% bei den Teilnehmerinnen zu rechnen. Neuere Daten zu Intervallkarzinomen aller schweizerischen Programme stehen derzeit leider noch nicht zur Verfügung. In Holland, das zu den Ländern mit sehr 


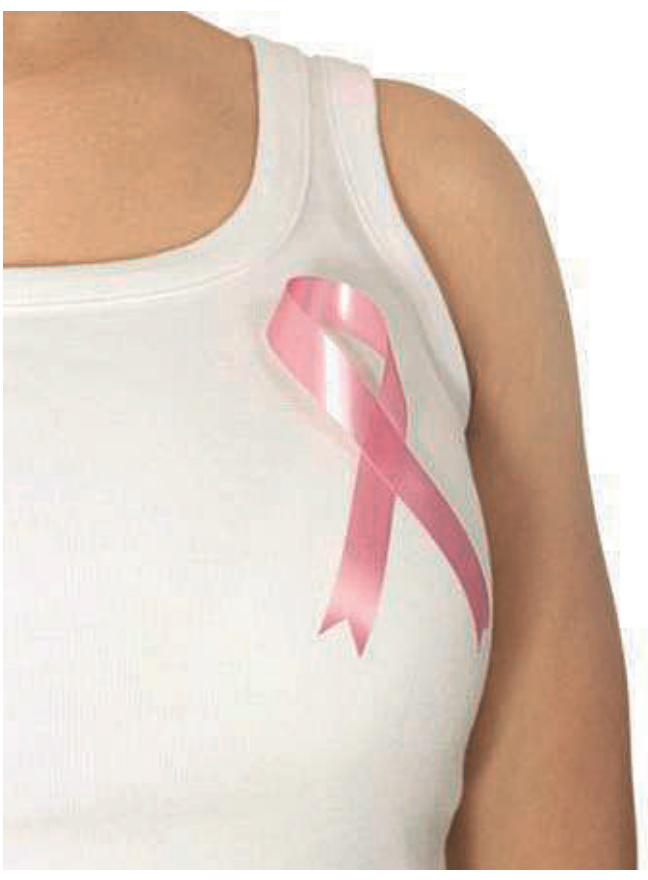

Bei der Qualitätsbeurteilung muss zwischen Prävalenzscreenings und Folgescreenings unterschieden werden.

hoher Brustkrebsinzidenz zählt, war die so errechnete Brustkrebsprävalenz im Mammographieprogramm der 2011 untersuchten Frauen mit 0,84\% (6,3 frühentdeckte Brustkrebse und 2,1 Intervallkarzinome je 1000 untersuchte Frauen) ebenfalls etwas niedriger als die von Ritschard vorausgesetzte Prävalenz von 1\% [9].

\section{In den meisten Bereichen wurden die Kennzahlen der europäischen Leitlinien zur Qualitätssicherung erreicht.}

Dass der Autor in Tabelle 3 sogar ein Szenario einer Prävalenz von 1,8\% heranzieht, ist nicht nachvollziehbar. Er basiert seine Annahme der Prävalenz auf einer Schätzung von Frauen, bei denen irgendwann - meist viele Jahre früher - eine Brustkrebsdiagnose gestellt worden war. Diese Frauen nehmen aus offensichtlichen Gründen gar nicht mehr am Screening teil. Zudem ist diese Schätzung aus dem Bericht «Krebs in der Schweiz» integral für alle Altersgruppen der Frauen, und die Hälfte dieser Brustkrebsüberlebenden dürfte über 70 Jahre alt sein [10].

Üblicherweise beruht die Beurteilung einer genügenden Screeningperformance auf dem Vergleich mit den $\mathrm{zu}$ erwartenden Brustkrebsinzidenzziffern, wenn es kein Screeningprogramm geben würde. In der Periode 1997-2001 wurde jährlich bei 2,8 pro 1000 in der Schweiz wohnenden Frauen im Alter von 50 bis 69 Jahren ein Brustkrebs diagnostiziert [11]. Die Europäischen Leitlinien zur Qualitätssicherung des Brustkrebsscree- nings [12] empfehlen, dass die Brustkrebsentdeckungsrate bei Erstscreenings mehr als dreimal so hoch und beim Folgescreening mehr als anderthalb mal so hoch sein soll wie die zu erwartende Inzidenzrate ohne Screening; allerdings ohne genauen Hinweis, wie diese Rate ohne Screening eruiert werden kann. Im Jahr 2011 lagen bei den Mammographieprogrammen in der Schweiz die Brustkrebsentdeckungsraten bei ErstScreenings etwa zwei- bis dreimal so hoch wie die Inzidenzziffer Ende der 90er Jahre und bei Folgescreenings mehr als anderthalbmal so hoch.

Der Anteil invasiver Karzinome mit einem Durchmesser von $15 \mathrm{~mm}$ oder weniger ist bei Folgescreenings tatsächlich höher als beim Erstscreening, wie Herr Ritschard zu Recht feststellt. Die vielleicht treffendere Feststellung wäre aber, dass demzufolge der Anteil der grösseren invasiven Karzinome beim Folgescreening wesentlich kleiner ist. Dies ist zu erwarten, weil davon ausgegangen werden kann, dass die im Prävalenzscreening entdeckten Brustkrebsfälle sich über einen variableren und längeren Zeitraum entwickelt hatten als die in den Folgescreenings entdeckten. Ein höherer Anteil der kleineren invasiven Tumoren in den Folgescreenings wird ebenfalls in den Europäischen Leitlinien erwartet (Tabelle 33, S. 46). Da ein initiales Screening allfällige Unterschiede der Frauen bezüglich früher durchgeführter Mammographien (etwa als opportunistisches Screening) abschwächt, sind generell die Kennzahlen für Folgescreenings besser für Vergleiche über Programme oder Länder geeignet. Korrekt ist allerdings die Feststellung, dass die Häufigkeit weiterer Abklärungen höher ist, als es die Europäischen Leitlinien empfehlen, und dass hier Verbesserungspotential besteht.

\section{Fazit}

Zusammenfassend stellen wir fest, dass die Berechnungen von Herrn Ritschard innerhalb seiner Annahmen und Szenarien kohärent durchgeführt wurden, dass diese aber nicht präzise zwischen Prävalenzscreenings und Folgescreenings unterscheiden, und dass die Zahlen des «internationalen Vergleichs» in Wahrheit keine solchen sind. Ein echter Vergleich mit den Europäischen Leitlinien zur Qualitätssicherung des Brustkrebsscreenings zeigt, dass in den meisten Bereichen die vorgegebenen Kennzahlen erreicht wurden, und dass die Qualität der Mammographieprogramme in der Schweiz insgesamt nicht als mangelhaft einzustufen ist. Es ist gerade die Stärke des Screenings in Programmen, dass diese regelmässig über die erreichten Resultate Rechenschaft ablegen. Dazu gehört auch, dass die Resultate kritisch diskutiert werden können. 


\section{Literatur}

1 Ritschard R. Mangelnde Qualität der MammographiescreeningProgramme? Schweiz Ärztezeitung. 2015;96(49):1823-5.

2 Bulliard JL, Zwahlen M, Fracheboud J. Swiss Cancer Screening. Dépistage par mammographie en Suisse, Année 2011. Berne: Swiss Cancer Screeninng 2014. www.swisscancerscreening.ch/ brustkrebs/fachinformationen/monitoring

3 Gigerenzer G, Kuoni J, Ritschard R. Was Ärzte wissen müssen. Schweiz Medizin-Forum. 2015;15(36):787-93.

4 Kooperationsgemeinschaft Mammographie. Evaluationsbericht 2005-2012. Ergebnis- und Prozessqualität im deutschen Mammographie-Screening-Programm. Berlin: Kooperationsgemeinschaft Mammographie; 2015.

5 Giordano L, Castagno R, Giorgi D, Piccinelli C, Ventura L, Segnan N, Zappa M. Breast cancer screening in Italy: evaluating key performance indicators for time trends and activity volumes. Epidemiologia \& Prevenzione. 2015;39(3)Suppl 1:30-9.

6 Lastier D, Salines E, Rogel A. Programme de dépistage du cancer du sein en France: résultats 2010, évolution depuis 2006. Saint-Maurice: Institut de veille sanitaire, 2013. (www.invs.sante.fr)

7 National Evaluation Team for Breast cancer screening in the Netherlands (NETB-LETB). National evaluation of breast cancer screening in the Netherlands 1990-2011/2012. Thirteen evaluation report NETB XIII. Rotterdam/Nijmegen: Department of Public Health, Erasmus University Medical Center Rotterdam and Department for Health Evidence, Radboud University Nijmegen Medical Centre; 2014

8 Bulliard JL, Ducros C, Dayer E, Arzel B, Levi F. Variation in performance in low-volume mammography screening programmes: experience from Switzerland. Cancer epidemiology. 2011;35:293-7.

9 National Evaluation Team for Breast cancer screening in the Netherlands (NETB-LETB). LETB/NETB Monitor 2013. Nation-wide breast cancer screening in the Netherlands. Results 1990-2013. Rotterdam/Nijmegen: Department of Public Health, Erasmus University Medical Center Rotterdam and Department for Health Evidence, Radboud University Nijmegen Medical Centre, 2015

10 Lorez M, Heusser R, Arndt V. Prevalence of Cancer Survivors in Switzerland. Schweizer Krebsbulletin. 2014:285-9.

11 www.bfs.admin.ch/bfs/portal/de/index/infothek/onlinedb/ stattab.html, accessed on 4 December 2015.

12 Perry N, Broeders M, de Wolf C, Törnberg S, Holland R, von Karsa L. European guidelines for quality assurance in breast cancer screening and diagnosis. Fourth Edition. Luxembourg: Office for Official Publications of the European Communities; 2006. S. 12. 